\title{
All-dielectric metamaterial frequency selective surface based on spatial arrangement ceramic resonators
}

\author{
Liyang $\mathrm{Li}^{*}$, Jun Wang ${ }^{\dagger}$, Mingde Feng, Hua Ma, Jiafu Wang, Hongliang Du and Shaobo Qu
} Science College, Air Force Engineering University, Xi'an 710051 Shaanxi, P. R. China

\author{
*yjszlzyyx@126.com \\ †wangjun563.163.com \\ tqushaobo@mail.xjtu.edu.cn
}

Received 22 January 2017; Accepted 1 March 2017; Published 29 March 2017

\begin{abstract}
In this paper, we demonstrate a method of designing all-dielectric metamaterial frequency selective surface (FSS) with ceramic resonators in spatial arrangement. Compared with the traditional way, spatial arrangement provides a flexible way to handle the permutation and combination of different ceramic resonators. With this method, the resonance response can be adjusted easily to achieve pass/stop band effects. As an example, a stop band spatial arrangement all-dielectric metamaterial FSS is designed. Its working band is in $11.65-12.23 \mathrm{GHz}$. By adjusting permittivity and geometrical parameters of ceramic resonators, we can easily modulate the resonances, band pass or band stop characteristic, as well as the working band.
\end{abstract}

Keywords: All-dielectric; metamaterial; FSS; ceramic resonators.

\section{Introduction}

Frequency selective surface (FSS) is a kind of periodic structures which are reflective or transparent for electromagnetic waves in certain frequency bands. FSS has been widely investigated over the past few decades. ${ }^{1-3} 2 \mathrm{D}$ periodic array of conducting patches/strips printed on dielectric substrate is a characteristic of the conventional FSS. Compared with metal, all-dielectric materials are hardly to breakdown in high voltage, also prevent themselves in oxidization and corrosion. All-dielectric materials are more suitable in highpower and high-temperature applications. With these advantages, all-dielectric FSSs come into being in recent years. Bertoni et al., ${ }^{4}$ Park et al. ${ }^{5,6}$ Magnusson et al. ${ }^{7-9}$ Bossard et al. ${ }^{10-12}$ and Barton et al. ${ }^{13,14}$ make pioneer works and great contribution to the developing of all-dielectric FSS. In our previous work, all-dielectric metamaterial stop band, ${ }^{15}$ pass band ${ }^{16}$ and reconfigurable ${ }^{17}$ FSS have been designed. In this work, we propose a method of designing three-dimensional (3D) spatially arranged all-dielectric metamaterial FSS. This FSS can easily modulate the resonant modes and working bands via tuning its parameters. Compared with 2D FSS, this FSS is more flexible to handle the permutation and combination. The method shows an efficient way to modulate the resonance to achieve pass/stop band effects.

\section{Modeling and Simulation}

The FSS is composed of cube ceramic resonators arranged in 3D space, as shown in Fig. 1. The basic unit cell is composed of four cubes arranged in space and periodically arranged in $x-o-y$ plane as shown in Fig. 1(a). In order to clearly illustrate the four cubes, we define the four cubes as specimen " 1 ", specimen "2", specimen "3" and specimen "4" as shown in Fig. 1(b). The four cubes are in the same size, $a$ ( $x$-direction) $=b(y$-direction $)=d(z$-direction $)=2 \mathrm{~mm}$. Figure $1(\mathrm{c})$ shows that the four cubes are at the same $y-O-z$ plane. Specimen " 1 " is $2 \mathrm{~mm}$ away from specimen "2" and specimen " 3 " in $z$-direction. Specimen " 2 " and specimen " 3 " are $2 \mathrm{~mm}$ away from specimen " 4 " in $z$-direction. Specimen " 2 " is $0.5 \mathrm{~mm}$ away from Specimen "1" and Specimen "4" in y-direction. Specimen "1" and Specimen "4" are $0.5 \mathrm{~mm}$ away from Specimen " 3 " in $y$-direction. Because the structure is composed of many spatial arrangement ceramics resonators, in order to realize the structure, we used 3D printer to fabricate a support as shown in Fig. 1(d). The support can hold all the ceramic resonators periodically in a 3D arrangement. On the other hand, the relative permittivity of the used ceramic is over 100. 3D printer materials we use is the polylactic acid whose relative permittivity is lower than 5 at the working band of the FSS. Thus, the support only has little influence on the FSS. The period of the unit cell is $P_{x}=4 \mathrm{~mm}$ and $P_{y}=8 \mathrm{~mm}$.

The ceramic we use is $0.7 \mathrm{Ba}_{0.6} \mathrm{Sr}_{0.4} \mathrm{TiO}_{3}-0.3 \mathrm{La}$ $\left(\mathrm{Mg}_{0.5} \mathrm{Ti}_{0.5}\right) \mathrm{O}_{3}$. The relative permittivity and the loss tangent of the ceramic are $\varepsilon_{r}=110$ and $\tan \delta=0.002$, respectively. The technological process of the ceramic is illustrated in Ref. 15. The frequency-domain solver of CST Microwave Studio is used for simulation. The unit cell boundary conditions are applied on the four boundaries along $x$ - and $y$-directions.

This is an Open Access article published by World Scientific Publishing Company. It is distributed under the terms of the Creative Commons Attribution 4.0 (CC-BY) License. Further distribution of this work is permitted, provided the original work is properly cited. 


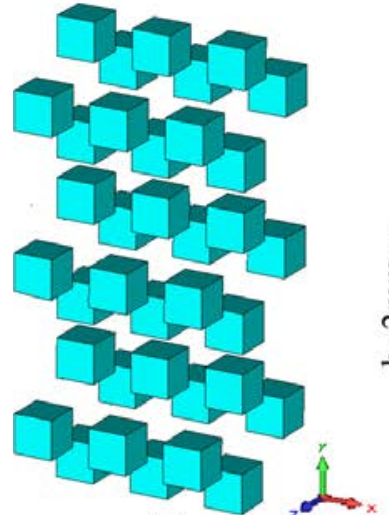

(a)

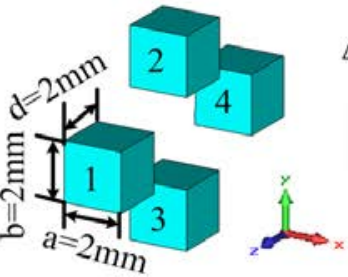

(b)

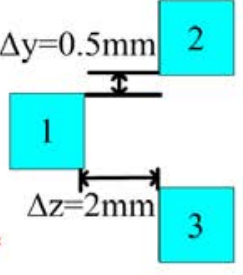

(c)

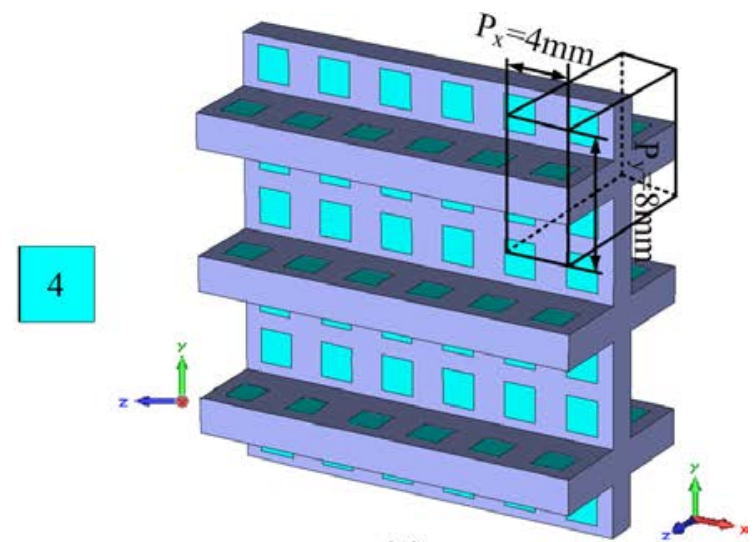

(d)

Fig. 1. The structure of the FSS.

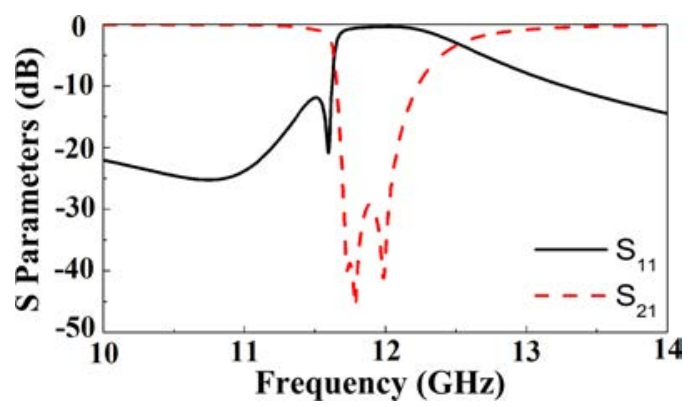

Fig. 2. The simulation results of the FSS.

Figure 2 shows the simulation results of the structure under normally incident $y$-polarized plane waves. It can be seen that there is a stop band in $11.65-12.23 \mathrm{GHz}$. The most stop band are under $-30 \mathrm{~dB}$, which demonstrates the stop band can work well.

\section{Analysis and Discussion}

It can be seen from Fig. 2 that there are three resonant points at $11.728,11.784$ and $11.988 \mathrm{GHz}$ in the stop band. In order to analyze the resonant of the structure, we observe the electric fields and magnetic fields of the resonant points. Dielectric resonator theory ${ }^{18-21}$ is also employed to analyze the structure. Because the structure is a 3D structure, it is difficult to describe the position of every cube. We define $\alpha$, $\beta, \gamma$, and $\delta$ planes as shown in Fig. 3. $\alpha$ plane is a $y-o-z$ plane on the middle of the structure in the $x$-direction. $\beta$ is a $x-o-y$ plane on the middle of Specimen " 1 " in the $z$-direction. $\gamma$ is a $x-O-y$ plane on the middle of Specimen " 2 " and Specimen " 3 " in the $z$-direction. $\delta$ is a $x-O-y$ plane on the middle of Specimen " 4 " in the $z$-direction.

The first resonant point is at $11.728 \mathrm{GHz}$. The second resonant point is at $11.784 \mathrm{GHz}$ and the third resonant point is at $11.988 \mathrm{GHz}$. From the electric fields at $11.728,11.784$ and $11.988 \mathrm{GHz}$, it can be seen that the resonant modes inside the four cubes are in the same form. There is an electric field loop forms on $y-O-z$ plane in the four cubes. The magnetic field inside each cube is resonant back and forth, which is equivalent to a magnetic dipole. This resonant mode corresponds to $\mathrm{TE}_{\delta 11}^{x}$ mode of the rectangular dielectric resonator, where the superscript $x$ means that the electric field is transverse with respect to $x$-direction and $0<\delta<1 .{ }^{15}$ The difference is the phase. It can be seen that at $11.728 \mathrm{GHz}$, the direction of

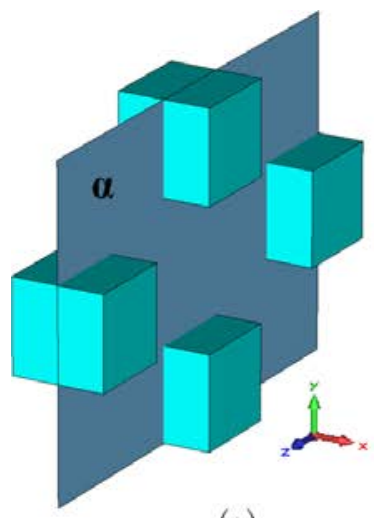

(a)

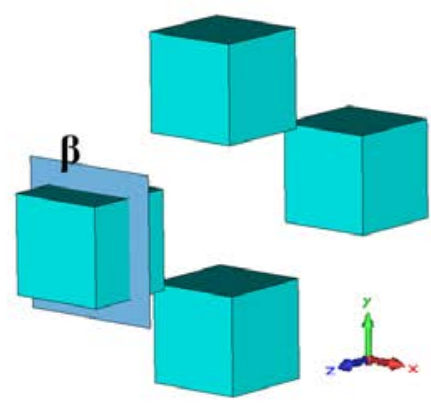

(b)

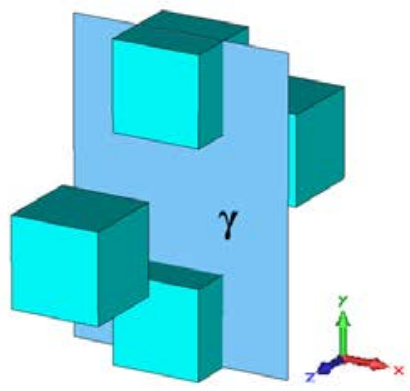

(c)

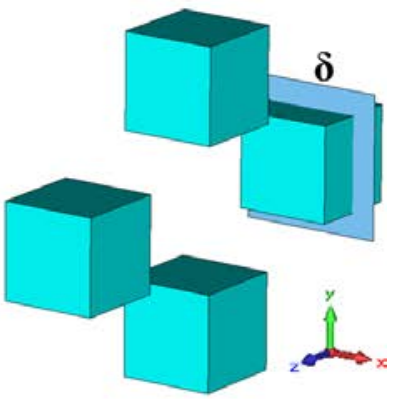

(d)

Fig. 3. The definition of $\alpha, \beta, \gamma$, and $\delta$ planes. 


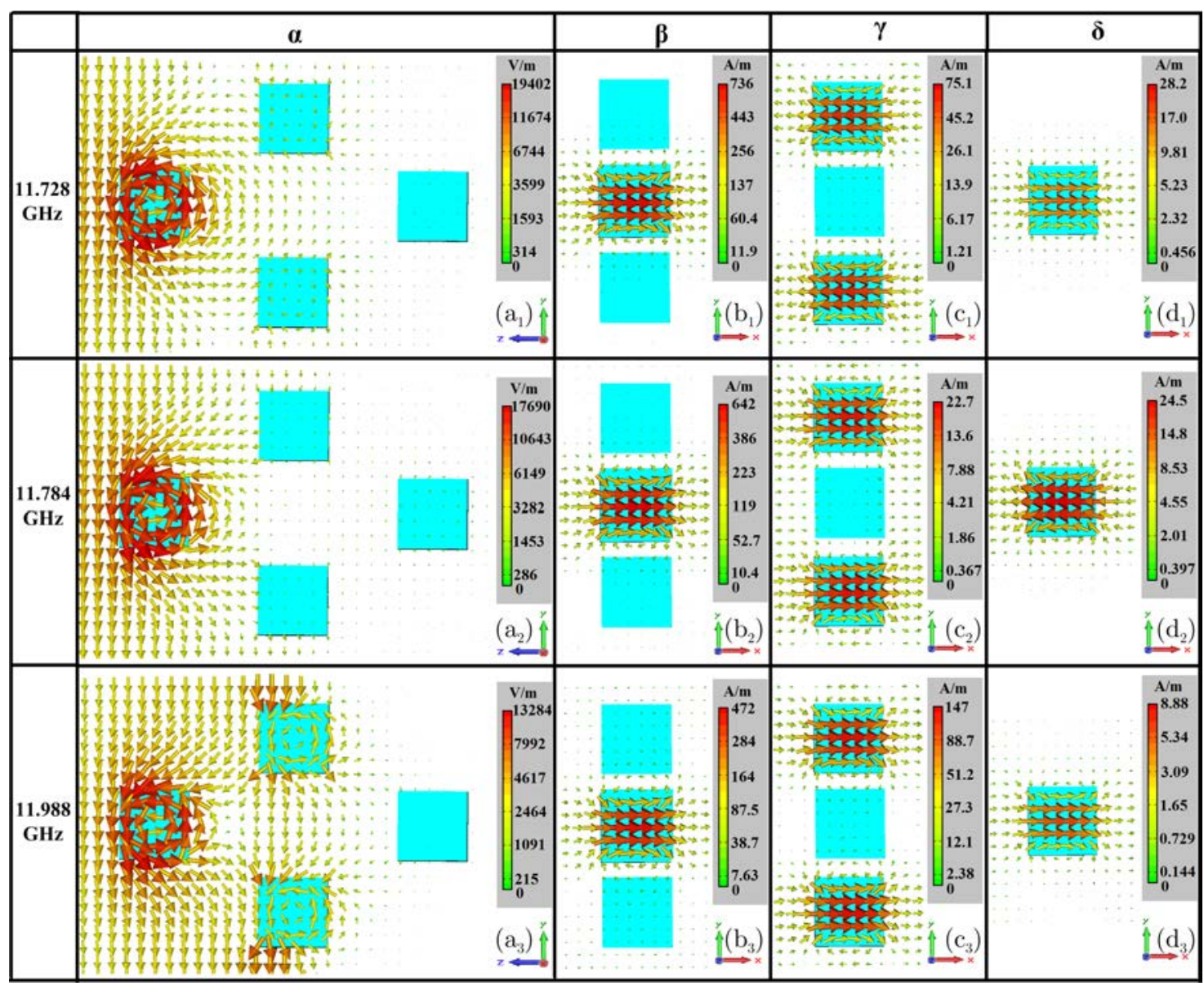

Fig. 4. The electric fields and magnetic fields of three resonant points.

magnetic resonance in Specimen "2" and Specimen "3" are different from Specimen "1" and Specimen "4". From the amplitude at the right of Figs. $4\left(b_{1}\right), 4\left(c_{1}\right)$ and $4\left(d_{1}\right)$, the intensity of resonance at Specimen "1" is the strongest. Although the resonances in Specimen "2" and Specimen "3" are opposite from Specimen "1" and Specimen "4", the main resonance is at Specimen "1". At $11.784 \mathrm{GHz}$, the resonance in Specimen "1", Specimen "2" and Specimen "3" are the same. The resonance in Specimen "4" is opposite from them. But the main resonance is still in Specimen "1". At $11.988 \mathrm{GHz}$, it can be seen that the resonance inside the four cubes are the same, although the strongest resonance is still in Specimen "1", the four cubes resonate in coordination. Because the sizes of four cubes are same and for normal incident $y$-polarized plane waves this structure is symmetrical, so it is easy to inspire the same resonant mode in the cubes.

The resonant modes can be modulated to tuning the working band. If we change the permittivity, $a, b$, or $d$, we can modulate the electromagnetic characteristics of the FSS. For example, first we keep permittivity, $a, P_{x}$ and $P_{y}$ stable. We change $b$ (y-direction) of Specimen " 1 " and Specimen "4" to $4.6 \mathrm{~mm}$, change $d$ (z-direction) of Specimen "2" and

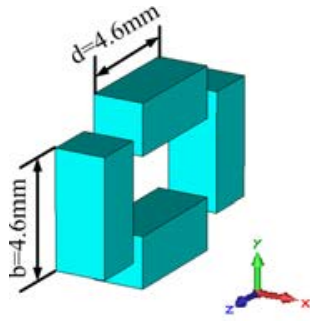

(a)

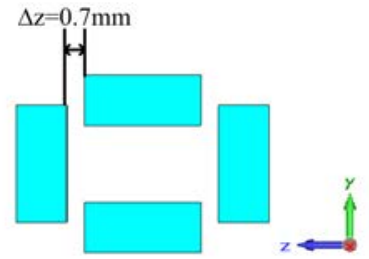

(b)

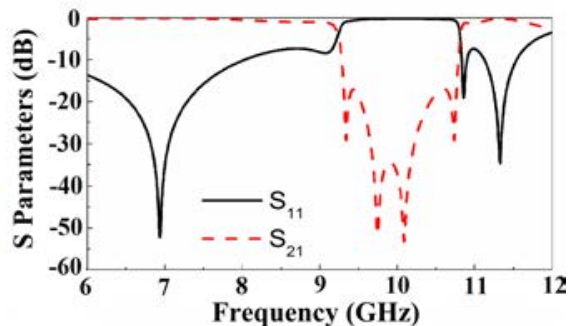

(c)

Fig. 5. Changed structure and its simulation results. 
Specimen "3" to $4.6 \mathrm{~mm} . \Delta z=0.7 \mathrm{~mm}$. Changed structure is shown in Figs. 5(a) and 5(b). The simulation results of the structure under normally incident $y$-polarized plane waves are shown in Fig. 5(c). It can be seen that changing $b$ and $d$ can result in the differences between the four ceramic resonators. In this case, the cubes becomes cuboids. The four ceramic resonators are not performed in the same way anymore, it destroy the balance of the four ceramics resonators at some phase angles. So it can inspire new resonant modes. At this time, the resonant modes in the four cubes are not the same any more. From the results, we can see that the stop band may become better. We can adjust some parameters of the structure to modulate the resonant modes, as well as tuning its electromagnetic responses.

\section{Conclusion}

In this paper, we demonstrate a method of designing alldielectric metamaterial FSS. The spatial arrangement method can provide a flexible way to handle the permutation and combination of different ceramic resonators. A simple spatial arrangement FSS is designed and works in $11.65-12.23 \mathrm{GHz}$. This FSS contains four same cube ceramics resonators, which inspires the same resonant mode to achieve the stop band. This kind of FSS is very easy to adjust its parameters such as $a, b, d$, permittivity to realize or optimize the FSS we need. Moreover, it can possess many arrangement forms in free space, which can inspire more resonant modes. With this method, the resonance response can be modulated easily to achieve pass/stop band effects.

\section{Acknowledgments}

The authors would like to thank the Natural Science Foundation of China (NSFC) through the grant nos. 11504428, 61671466 and 11274389, the Natural Science Foundation of Shaanxi Province under Grant 2016JM6026.

\section{References}

${ }^{1}$ B. A. Munk, Frequency-Selective Surfaces: Theory and Design (Wiley, New York, 2000).

${ }^{2}$ T. K. Wu, Frequency Selective Surfaces and Grid Arrays (Wiley, New York, 1995).

${ }^{3}$ E. Pelton and B. Munk, A streamlined metallic radome, IEEE Trans. Antennas Propag. 22, 799 (1974).

${ }^{4}$ H. L. Bertoni, L. S. Cheo and T. Tamir, Frequency-selective Reflection and transmission by a periodic dielectric layer, IEEE Trans. Antennas Propag. 37, 78 (1989).
${ }^{5}$ D. C. Park, G. L. Matthaei and M. S. Wei, Dielectric waveguide grating design for bandstop and bandpass filter applications, IEEE MTT-S Int. Microwave Symp. (1984), pp. 202-204.

${ }^{6}$ D. C. Park, G. L. Matthaei and M. S. Wei, Bandstop filter design using a dielectric waveguide grating, IEEE Trans. Microw. Theory Tech. 33, 693 (1985).

${ }^{7}$ R. Magnusson, S. S. Wang, T. D. Black and A. Sohn, Resonance properties of dielectric waveguide gratings: Theory and experiments at $4-18 \mathrm{GHz}$, IEEE Trans. Antennas Propag. 42, 567 (1994).

${ }^{8}$ R. Magnusson and S. S. Wang, Transmission bandpass guidedmode resonance filters, Appl. Opt. 34, 8106 (1995).

${ }^{9}$ S. Tibuleac, R. Magnusson, T. A. Maldonado, D. Shin and C. Zuffada, Direct and inverse techniques of guided-mode resonance filters designs. IEEE AP-S Int. Symp., Vol. 4 (1997), pp. 2380-2383.

${ }^{10} \mathrm{~J}$. A. Bossard et al., Terahertz applications of frequency selective surfaces: Analysis, design, fabrication and testing, IEEE AP-S Int. Symp. (2006), pp. 2395-2398.

${ }^{11}$ J. A. Bossard et al., Multiband all-dielectric frequency selective surface filters for the mid-infrared, IEEE AP-S Int. Symp. (2007), pp. 3416-3419.

${ }^{12}$ S. Yun, J. A. Bossard, T. S. Mayer and D. H. Werner, Angle and polarization tolerant midinfrared dielectric filter designed by genetic algorithm optimization, Appl. Phys. Lett. 96, 223101 (2010).

${ }^{13} \mathrm{~J}$. H. Barton et al., All-dielectric frequency selective surface for high power microwaves, IEEE Trans. Antennas Propag. 62, 3652 (2014).

${ }^{14}$ J. H. Barton, C. R. Garcia, E. A. Berry, R. Salas and R. C. Rumpf, 3-D printed all-dielectric frequency selective surface with large bandwidth and field of view, IEEE Trans. Antennas Propag. 63, 1032 (2015)

${ }^{15}$ L. Y. Li et al., All-dielectric metamaterial frequency selective surfaces based on high-permittivity ceramic resonators, Appl. Phys. Lett. 106, 212904 (2015).

${ }^{16}$ L. Y. Li et al., Achieving all-dielectric metamaterial band-pass frequency selective surface via high permittivity ceramics, Appl. Phys. Lett. 108, 122902 (2016).

${ }^{17} \mathrm{~L}$. Y. Li et al., Reconfigurable all-dielectric metamaterial frequency selective surface based on high-permittivity ceramics, Sci. Rep. 6, 24178 (2016).

${ }^{18}$ J. Wang, Z. Xu, Z. H. Yu, X. Y. Wei, Y. M. Yang, J. F. Wang and S. B. Qu, Experimental realization of all-dielectric composite cubes/rods left-handed metamaterial, J. Appl. Phys. 109, 084918 (2011).

${ }^{19}$ B. Du, J. Wang, Z. Xu, S. Xia, J. F. Wang and S. B. Qu, Band split in multiband all-dielectric left-handed metamaterials, J. Appl. Phys. 115, 234104 (2014).

${ }^{20}$ Y. M. Yang, J. F. Wang, S. Xia, P. Bai, Z. Li, J. Wang, Z. Xu and S. B. Qu, All-dielectric left-handed metamaterial based on dielectric resonator: Design, simulation and experiment, Chin. Phys. B 20, 014101 (2011)

${ }^{21}$ K. M. Luk and K. W. Leung, Dielectric Resonator Antennas, Chap. 11 (Research Studies Press LTD, Baldock, Hertfordshire, England, 2003). 